METHODS-INEMOLECULAR MEDI CINE TM Pancreatic
Cancer. 13

\title{
Methods and Protocols
}

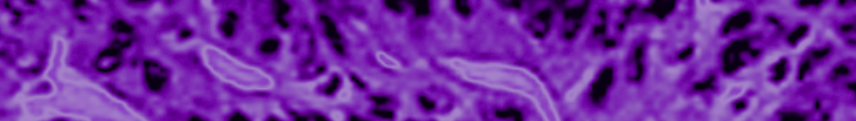

Edited by

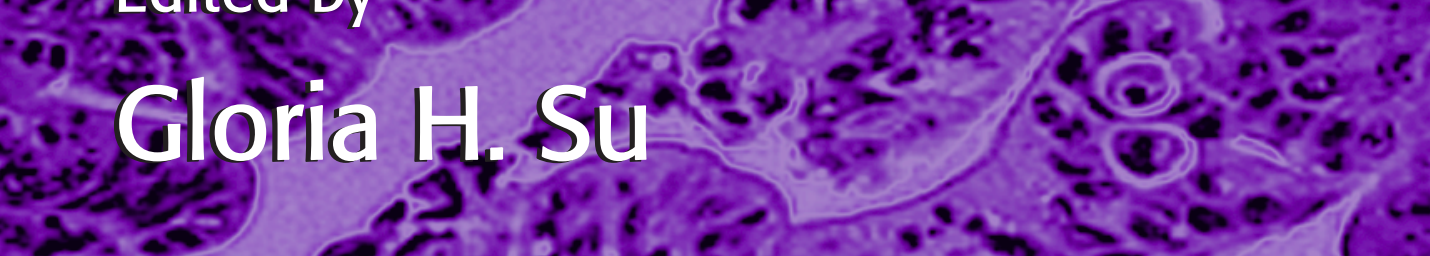
सtते 109 26. Cow

8

4. HuMANAPRESS 


\title{
Practical Methods for Tissue Microarray Construction
}

\author{
Helen L. Fedor and Angelo M. De Marzo
}

\section{Summary}

The tissue microarray (TMA) of Kononen et al. is an extension of an idea originally developed by Battifora and consists of an array of cylindrical cores of paraffin-embedded tissue that are removed from preexisting "donor" paraffin blocks. The donor block is a standard tissue block that may be from surgical pathology, autopsy, or research material. A morphologically representative area of interest within the donor block is identified under the microscope using a stained section (usually hematoxylin and eosin stained) on a glass slide as a guide. The tissue cores are removed from the donor and inserted into a "recipient" paraffin block usually using a custom patented instrument from Beecher Instruments. Using a precise spacing pattern, tissues are inserted at high density, with up to 1000 tissue cores in a single paraffin block. Sections from this block that are cut with a microtome are placed onto standard slides that can then be used for in situ analysis. Depending on the overall depth of tissue remaining in the donor blocks, tissue arrays can generate between 100 and 500 sections. Once constructed tissue microarrays can be used with a wide range of techniques including histochemical staining, immunohistochemical/immunofluorescent staining, or in situ hybridization for either DNA or mRNA. In this chapter we present methods of TMA construction with emphasis on providing detailed tips and techniques.

Key Words: Tissue microarrays; image analysis; bioinformatics; immunohistochemistry.

\section{Introduction}

Tissue microarrays (TMAs) are emerging as a breakthrough in our ability to analyze rapidly the expression of existing and new biomarkers using archival pathology specimens. Multitissue blocks were first introduced by Battifora et al. in the so-called "sausage" or Multi-Tissue Tumor Block (MTTB), where up to 100 separate tissues were processed together into a single paraffin block (1). Recently, Kononen et al. introduced a new method of combining multiple tissues into a single paraffin block that uses a novel sampling approach with regular size and shaped tissues. This allows for many more specimens to be precisely 
arrayed into a single paraffin block (2). Several sources of information are available for tissue microarray protocols, tips, techniques, and troubleshooting. These include recent reviews (3-7), a detailed web site with protocols (http:// www.yalepath.org/DEPT/research/YCCTMA/YTMA_protocol.pdf), and sites developed by the NIH (http://resresources.nci.nih.gov/tarp/; http://www.nhgri. nih.gov/DIR/CGB/TMA).

Tissue microarray construction entails several key aspects that will be presented as an overview here, with details found in Subheading 3. These aspects include the following: (1) purpose of the TMA; (2) TMA design; (3) selection of appropriate tissue blocks; (4) identifying regions of interest within donor tissue blocks; (5) data handling; (6) array construction; (7) TMA block sectioning; (8) TMA slide staining; and (9) image handling (optional). In this chapter we present detailed methods of TMA construction, using our experience constructing more than 320 TMAs containing more than 45,000 tissue cores as a guide. In depth information regarding array construction and troubleshooting is also available form the Beecher Instruments website (http://www.beecher instruments.com/index.html) and instruction manual.

\subsection{Purpose of the TMA}

There are unlimited types of TMAs that may be of use depending on the project at hand. We have found that very useful arrays can be constructed from an assortment of normal human tissues obtained as discarded material from surgical specimens. These arrays are ideal for working up new antibodies or new probes for in situ hybridization. A second general type of array is one with a small number of diseased tissues from several patients. For example, when evaluating a new antibody for prostate cancer we often use an array with 20 cases of tumor and normal to quickly assess whether the marker appears of interest. A more sophisticated TMA can be constructed containing samples from many more patients (hundreds or more), and this may consist of a set of several individual TMA blocks. The true power of this type of approach is to correlate staining results with clinical outcome, if available. If possible, it is always advisable to include some matched normal tissue from which the tumor or other diseased tissue is derived. For our prostate cancer arrays, we provide a matched normal appearing epithelial sample from each sample of cancer.

\subsection{TMA Design}

Depending on the purpose of the TMA, the design may vary greatly. There are no established guidelines for TMA design. For pancreatic cancer, in which the prediction of clinical outcome is often not an important issue, the main purpose in constructing a TMA is to simply assemble a convenient series of 
Table 1

Typical Core Spacing and Number of Cores Using Various Needle Sizes

\begin{tabular}{lccc}
\hline Needle size & Spacing between samples & Array format & Total number of cores \\
\hline $0.6 \mathrm{~mm}$ & $0.2 \mathrm{~mm}$ & $20 \times 20$ cores & 400 \\
$1.0 \mathrm{~mm}$ & $0.3 \mathrm{~mm}$ & $16 \times 13$ cores & 208 \\
$1.5 \mathrm{~mm}$ & $0.4 \mathrm{~mm}$ & $11 \times 9$ cores & 99 \\
\hline
\end{tabular}

patients into one or a few TMA blocks. For example, we have produced arrays of invasive pancreatic adenocarcinoma, pancreatic intraepithelial neoplasia, intraductal pancreatic mucinous neoplasms, and so forth. These arrays typically contain between 50 and 100 patient samples.

One key consideration is the size of the punch. Four sizes are available: 0.6 $\mathrm{mm}, 1.0 \mathrm{~mm}, 1.5 \mathrm{~mm}$, and $2.0 \mathrm{~mm}$. Although some investigators prefer the 2.0$\mathrm{mm}$ size, these large cores may damage the donor and recipient blocks (see Note 1). If larger cores are desired, the $1.5-\mathrm{mm}$ core may be acceptable, as it seems to cause less damage but still covers a large area. The $1.5-\mathrm{mm}$ core is the preferred core size of most of the pancreatic cancer arrays that have been constructed at our institution, as pancreatic cancer can be so sclerotic and tumor cells are often widely dispersed. For the majority of TMAs in our laboratory, however, the $0.6-\mathrm{mm}$ are used, as many studies have shown that one to four cores of this size from a given tissue yield as much information as standard tissue sections (8-12). The number of cores that can be put into a single TMA block is another important consideration. The reported record number of cores is 1200. But it is usual not to place more than 600 (see Note 2).

The spacing between samples is the next consideration. Spaces from 0.1 $\mathrm{mm}$ and higher have been used successfully (see Note 3). Table 1 shows a convenient spacing pattern between cores and the number of cores that can be put into the array block without rotating the block during construction.

The array is usually laid out in an electronic spreadsheet. Figure 1 shows a sample "map" used to design and construct an example array utilizing the 0.6$\mathrm{mm}$ punch. The resulting array will have 10 cores across ( $x$-axis) and 6 cores down ( $y$-axis). This TMA is designed to have four replicate cores from each of the sample areas (individual tissue diagnoses), with the tumor and normal tissues from each case in the same row. All pertinent information that identifies a sample should be included in the map design.

In most of our TMAs we place control normal tissues or cell lines in strategic regions throughout the blocks. Any kind of control tissue may be used: normal tissues, cell lines, or animal tissue (see Note 4). Often, we place entire columns of various control tissues between the tumor and normal tissue, or 


\begin{tabular}{|c|c|c|c|c|c|c|c|c|c|c|c|}
\hline \multirow[b]{2}{*}{ Case } & \multicolumn{4}{|c|}{ TUMOR } & \multirow{3}{*}{$\begin{array}{c}\text { CONTROL } \\
\text { Liver }\end{array}$} & \multicolumn{4}{|c|}{ NORMAL } & \multirow{4}{*}{$\begin{array}{c}\text { CONTROL } \\
\text { Kidney }\end{array}$} & \multirow[b]{2}{*}{0} \\
\hline & \multirow{4}{*}{$\begin{array}{c}\text { Case } 1 \\
\text { Block B } \\
\text { A } \\
01,01\end{array}$} & \multirow{4}{*}{$\begin{array}{c}\text { Case } 1 \\
\text { Block B } \\
\text { A } \\
02,01\end{array}$} & \multirow{4}{*}{$\begin{array}{c}\text { Case } 1 \\
\text { Block B } \\
\text { A } \\
03,01\end{array}$} & \multirow{4}{*}{$\begin{array}{c}\text { Case 1 } \\
\text { Block B } \\
\text { A } \\
04,01\end{array}$} & & \multirow{4}{*}{$\begin{array}{c}\text { Case } 1 \\
\text { Block B } \\
\text { B } \\
06,01\end{array}$} & \multirow{4}{*}{$\begin{array}{c}\text { Case 1 } \\
\text { Block B } \\
\text { B } \\
07,01\end{array}$} & \multirow{4}{*}{$\begin{array}{c}\text { Case } 1 \\
\text { Block B } \\
\text { B } \\
08,01\end{array}$} & \multirow{4}{*}{$\begin{array}{c}\text { Case 1 } \\
\text { Block B } \\
\text { B } \\
09,01\end{array}$} & & \\
\hline Block & & & & & & & & & & & \\
\hline Tissue Diagnosis & & & & & & & & & & & \\
\hline Coordinates & & & & & 05,01 & & & & & 10,01 & \\
\hline Case & Case 2 & Case 2 & Case 2 & Case 2 & \multirow{3}{*}{ Skin } & \multirow{4}{*}{$\begin{array}{c}\text { Case } 2 \\
\text { Block D } \\
\text { B } \\
06,02\end{array}$} & \multirow{4}{*}{$\begin{array}{c}\text { Case } 2 \\
\text { Block D } \\
\text { B } \\
07,02\end{array}$} & \multirow{4}{*}{\multicolumn{2}{|c|}{$\begin{array}{cc}\text { Case 2 } & \text { Case 2 } \\
\text { Block D } & \text { Block D } \\
\text { B } & \text { B } \\
08,02 & 09,02\end{array}$}} & \multirow{3}{*}{ Tonsil } & \multirow[t]{4}{*}{0.8} \\
\hline Block & Block A & Block A & Block A & Block A & & & & & & & \\
\hline Tissue Diagnosis & C & C & C & C & & & & & & & \\
\hline Coordinates & 01,02 & 02,02 & 03,02 & 04,02 & 05,02 & & & & & 10,02 & \\
\hline Case & Case 3 & Case 3 & Case 3 & Case 3 & \multirow{3}{*}{ Brain } & \multirow{4}{*}{$\begin{array}{c}\text { Case } 3 \\
\text { Block C } \\
\text { A } \\
06,03\end{array}$} & \multirow{4}{*}{$\begin{array}{c}\text { Case } 3 \\
\text { Block C } \\
\text { A } \\
07,03\end{array}$} & \multirow{4}{*}{$\begin{array}{c}\text { Case } 3 \\
\text { Block C } \\
\text { A } \\
08,03\end{array}$} & \multirow{4}{*}{$\begin{array}{c}\text { Case } 3 \\
\text { Block C } \\
\text { A } \\
09,03\end{array}$} & \multirow{3}{*}{ Muscle } & \multirow[t]{2}{*}{1.6} \\
\hline Block & Block K & Block K & Block K & Block K & & & & & & & \\
\hline Tissue Diagnosis & A & A & A & A & & & & & & & \\
\hline Coordinates & 01,03 & 02,03 & 03,03 & 04,03 & 05,03 & & & & & 10,03 & \\
\hline Case & Case 4 & Case 4 & Case 4 & Case 4 & \multirow{3}{*}{ Kidney } & Case 4 & Case 4 & Case 4 & Case 4 & \multirow{3}{*}{ Pancreas } & 2.4 \\
\hline Block & Block G & Block G & Block G & Block G & & Block G & Block G & Block G & Block G & & \\
\hline Tissue Diagnosis & B & B & B & B & & A & A & A & A & & \\
\hline Coordinates & 01,04 & 02,04 & 03,04 & 04,04 & 05,04 & 06,04 & 07,04 & 08,04 & 09,04 & 10,04 & \\
\hline Case & Case 5 & Case 5 & Case 5 & Case 5 & & Case 5 & Case 5 & Case 5 & Case 5 & & 3.2 \\
\hline Block & Block F & Block F & Block F & Block F & Stomach & Block T & Block T & Block T & Block T & Prostate & \\
\hline Tissue Diagnosis & A & A & A & A & & A & A & A & A & & \\
\hline Coordinates & 01,05 & 02,05 & 03,05 & 04,05 & 05,05 & 06,05 & 07,05 & 08,05 & 09,05 & 10,05 & \\
\hline Case & Case 6 & Case 6 & Case 6 & Case 6 & & Case 6 & Case 6 & Case 6 & Case 6 & & 4 \\
\hline Block & Block M & Block M & Block M & Block M & Colon & Block N & Block N & Block N & Block N & Breast & \\
\hline Tissue Diagnosis & B & B & B & B & & C & C & C & c & & \\
\hline Coordinates & 01,06 & 02,06 & 03,06 & 04,06 & 05,06 & 06,06 & 07,06 & 08,06 & 09,06 & 10,06 & \\
\hline $\begin{array}{c}\text { Distances Between } \\
\text { Punches }\end{array}$ & 0 & 0.8 & 1.6 & 2.4 & 3.2 & 4 & 4.8 & 5.6 & 6.4 & 7.2 & \\
\hline
\end{tabular}

Fig. 1. Example of spreadsheet containing TMA core data.

asymmetrically at one end of the block. It is quite important to never construct an array block with complete symmetry, as one can lose orientation. For cell lines we fix cells in $10 \%$ neutral buffered formalin at room temperature overnight and embed them in $1 \%$ agarose prepared in phosphate-buffered saline (13). These cell plugs are then processed into paraffin blocks just as if they were pieces of tissue, making them available as donor blocks for TMAs.

Navigating a stained TMA slide can be cumbersome in that it is easy to lose one's place regarding the $x$ - and $y$-coordinates. Building in an orientation marker that indicates where the array begins can be very helpful. As first shown to our group by the M. A. Rubin laboratory (now at Harvard University), we use five tissue cores arranged in a plus sign (+), three cores placed vertically intersecting three cores placed horizontally. This is positioned in the upper left hand corner of the block. Although many TMA laboratories prefer to group array cores in separate regions of the blocks, we do not do this, as this can hamper automated image acquisition, at least on some systems.

\subsection{Tissue Selection}

The selection and collection of tissue blocks to be included in TMAs is the most time-consuming aspect of the entire project. Appropriate samples from clinical specimens are identified from the pathology archives of the given insti- 
tution. Glass slides corresponding to the entire cases for surgical pathology specimens are retrieved and reviewed to select appropriate candidate blocks (see Notes 5 and 6). Corresponding blocks are then obtained from the tissue archives. The area to be sampled, which usually represents a region corresponding to a specific pathological diagnosis ("tissue diagnosis") (14) or relevant normal tissue, is circled with a Pilot Pen (or similar xylene-free pen) by a pathologist or highly trained technician. Because the block may contain more than one tissue diagnosis of interest for obtaining tissue cores for a TMA, each circled tissue diagnosis on the slide is also assigned a letter or number.

\subsection{TMA Construction}

Details of array construction and TMA block sectioning are presented below. We recommend that you familiarize yourself with the equipment by constructing a practice array. The first couple of array blocks made will be far from perfect. By the third, expert status should be achieved (see Notes 7 and 8 ).

\subsection{TMA Block Sectioning}

Sectioning an array can be accomplished using standard sectioning procedures, although it is highly recommended to use a dedicated microtome. Prior to use the recipient blocks are faced off on this same microtome. In this fashion the amount of realignment of the microtome block holder is minimized. All the array blocks produced in the laboratory will have the same orientation, maximizing the number of slides that an array will yield (see Note 9). Changing blades frequently is advisable (see Note 10). Several investigators use the "Paraffin Sectioning Aid System" from Instrumedics Inc. This is called the Tape Transfer Method. Because we are still in the process of perfecting this method (see Notes 11 and 12), we refer the reader to the Instrumedics website (http://www.instrumedics.com).

\subsection{TMA Slide Staining}

TMA slides can be stained with any stain that can be used for standard slides, with out any modifications (see Notes 11 and 12).

\subsection{TMA Slide Data Handling}

In terms of TMA data handling, many investigators simply view the slides under a microscope and record their observations for each spot on paper or directly into a spreadsheet. Although presentation of detailed methods of TMA data handling are beyond the scope of this chapter, it should be pointed out that several highly specialized systems are in place and under development to store TMA data, as well as to scan TMA slides using automated imaging acquisition and to either score the images on the computer screen, or to have the system 
perform automated image analysis (see refs. 14-16 and http://www.pathology. pitt.edu/apiii02/Sci-DeMarzo.htm). The method of data storage and retrieval differs somewhat in the different systems. For example, data can either be entered into a spreadsheet (15), where they can be reformatted suitably for hierarchical clustering analysis or other statistical analysis, or be directly entered into a relational database (14; http://www.pathology.pitt.edu/apiii02/Sci-DeMarzo.htm), in which it too can be exported in a form suitable for statistical or other analysis. Demonstrations of some of these systems, in which the noncommercial software components can be obtained, are available online (http://tmaj.pathology. jhmi.edu; http://genome-www.stanford.edu/TMA/explore.shtml).

\section{Materials}

1. Manual tissue arrayer (Beecher Instruments, Sun Prarie, WI).

2. Tissue Array punches, sizes $0.6 \mathrm{~mm}, 1.0 \mathrm{~mm}, 1.5 \mathrm{~mm}$ (Sun Prarie, WI).

3. Paraplast X-tra (Fisher Scientific, Suwanee, GA, cat. no. 23-021-401).

4. Cap gap slides (Fisher Scientific, Suwanee, GA, cat. no. 12-548-6A).

5. Oven (Fisher Scientific, Suwanee, GA, cat. no. 11-695-1).

6. Magnifier on stand with attached light (Fisher Scientific, Suwanee, GA, cat. no. $8-882)$.

7. Stainless steel molds, $6 \mathrm{~mm}$ (Allegiance, Columbia, MD, cat. no. M7300-4).

8. Tissue cassettes (Allegiance, Columbia MD, cat. no. TN045).

9. Floatation water bath (Allegiance, Columbia MD, cat. no. M7654-1).

10. Accu Edge blades (Allegiance, Columbia, MD, cat. no. M7321-41).

11. Pilot Pen ultrafine point (Register Office Supply, Baltimore MD, cat. nos. 44104, 44103, and 44102 red, blue, and black).

12. Automated rotary microtome (Leica, Deerfield, IL, cat. no. RM2155).

\section{Methods}

Although a semiautomated tissue microarrayer is now available from Beecher Instruments, all the methods presented here involve the manual array system.

\subsection{Tissue Selection and Donor Block Preparation}

1. Identify the tissue of choice using a standard tissue section on a glass slide stained with hematoxylin and eosin (H\&E). An immunostained slide may also be employed (see Notes 5 and 6).

2. Circle the area to be sampled directly on the glass slide, which usually represents a region corresponding to a specific pathological diagnosis ("tissue diagnosis") (14) or relevant normal tissue, with a Pilot Pen (or similar xylene-free pen). Assign each separate region (tissue diagnosis) its own number or letter, such that it can be uniquely identified given the case number, block designation, and tissue diagnosis designation. 
3. Overlay the circled H\&E glass slide and the area of interest and identify the corresponding region on the block and circle with a laboratory marker (see Note 13).

4. The wax is melted at $56-59^{\circ} \mathrm{C}$ and then poured into a deep mold. A standard tissue cassette is placed on top (see Note 14).

5. Allow the block to chill completely at room temperature. After cooling, separate the cassette and mold.

\subsection{Equipment Setup for TMA Construction}

1. Each block and slide pair should be arranged in the order that they will be used following the map design.

2. Place the recipient block into the base plate and position the base plate on the arrayer (see Note 9).

3. Position a pair of punches in the arrayer (see Note 15). The larger one is used for extracting tissue from the donor block and is placed in the right punch holder (for right-handed persons). The smaller one is for making a hole in the recipient block where the donor tissue will be positioned, and is placed in the left punch holder (use the opposite configuration for left handed persons; see Note 16).

4. To ensure the alignment of the punches, first move the recipient punch into position and make a mark in the paraffin. Then do the same for the donor punch. These marks should coincide precisely (see Notes $\mathbf{1 7}$ and 18).

5. Move the needles to the position of the first punch with the $x$ - and $y$-axis micrometer adjustment knobs. The position of the punches over the block can be assessed by gently pushing down on them until a mark is made in the paraffin, continuing to make adjustments with the micrometer knobs until the desired position is attained.

6. Zero the micrometers.

\subsection{Array Construction (Assuming 0.6- $\mathrm{mm}$ Punches)}

At this point it may be necessary to adjust the depth stop, which dictates how deep the punch will be in the recipient block. Tightening or loosening the nut at the top left of the turret accomplishes this. The nut stops the downward movement of the turret to achieve the desired depth (see Note 19).

1. Apply pressure to the top of the turret to bring down the needle. A squeezing motion is used to push the tissue core into the paraffin.

2. Rotate the arm of the punch to the left and then back to the right, while maintaining pressure on the turret top. This rotating motion helps free the core from the recipient paraffin block (see Notes 20 and 21).

3. Release the pressure on the turret, allowing the springs to raise the turret to its resting position.

4. Press down on the stylus to eject the paraffin core from the punch and examine its length (see Note 22).

5. Make any necessary adjustment with the depth stop nut.

6. Swing the turret to the right to allow the donor needle to be brought into proper position. 


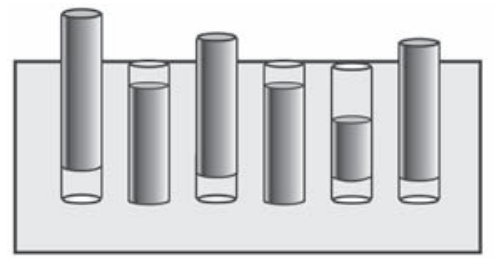

A Improperly placed cores

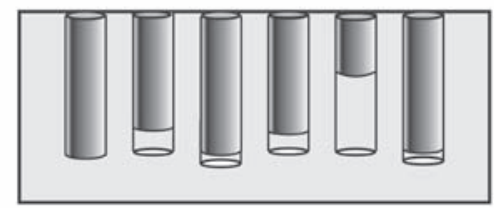

B Properly placed cores

Fig. 2. Correct and incorrect core placement. The placement of the core into the recipient block is the most challenging part of array construction. (A) Incorrect placement of tissue cores in a recipient block. (B) Correct placement. Tissue protruding from the surface of the block can be gently pressed down with a clean glass slide, until it becomes flush with the surface.

7. Place the bridge over the recipient block.

8. The donor block with the area of interest circled is placed on the bridge under the needle and a punch is taken from the inscribed sample area by repeating the procedure for removing the paraffin core from the recipient block (see Notes 1, 19, 21, 23, and 24).

9. The bridge and donor block are removed.

10. The core is inserted into the previously made hole by bringing the turret down until the lower punch surface is directly over the hole that was just created.

11. Keep the pressure on the turret and slowly push down on the stylus, guiding the core into the hole while expelling the tissue from the punch. This sample will have the coordinates 01, 01 from the map. See Fig. 2 for examples of proper and improper core placement (see Notes 20, 25-28).

12. Move the $x$-axis micrometer knob to $0.8 \mathrm{~mm}$ and repeat (see Notes 22, 29, and 30).

13. Continue across the row until the last core has been placed.

14. Now use the $x$-axis micrometer knob to go back to position zero and move the $y$ axis knob to $0.8 \mathrm{~mm}$, and repeat until the block is complete (see Notes $\mathbf{3 1}$ and $\mathbf{3 2}$ ).

\subsection{TMA Block Sectioning}

1. On completion of the array, remove it from the base plate and place into a $37^{\circ} \mathrm{C}$ oven for $15 \mathrm{~min}$ face down on a clean glass slide. This facilitates the adherence of the cores to the walls of the punches in the recipient block.

2. Remove the slide/block combination from the oven and apply gentle and even pressure. This evens out any irregularities in the block surface and removes the bulging of the block center that occurs during array construction (see Note 33).

3. Immediately place the TMA block onto a block of ice and wait until the complex is completely cooled before disassembly.

4. Set the temperature in the water bath to $30^{\circ} \mathrm{C}$. Section blocks at $4-5 \mu \mathrm{m}$, always placing sections on the slide in the same orientation (see Notes 34 and 35). 
5. Dry the slides overnight in a vertical position.

6. After drying slides place slides front to back and put into a stack, wrap tightly with Parafilm, label, and then store at $-20^{\circ} \mathrm{C}$ (see Note 36).

\subsection{Preparation of Cell Culture Blocks as TMA Block Donors}

1. Grow cells using your preferred method, depending on the cell line.

2. When cell cultures are 50-75\% confluent, detach cells and resuspend in $10 \%(\mathrm{v} / \mathrm{v})$ phosphate-buffered formalin at room temperature. The time of fixation can vary depending on the application; we typically fix over night.

3. Pellet fixed cells by centrifugation at $500 \mathrm{~g}$ for $10 \mathrm{~min}$, wash once in $1 \mathrm{X}$ PBS, and pellet again.

4. Resuspend cell pellets in an equal volume of $0.8 \%$ agarose (prepared in $1 \mathrm{X}$ PBS) at $42^{\circ} \mathrm{C}$.

5. Prefill the tapered end of a $0.6-\mathrm{mL}$ microfuge tube with agarose and let solidify.

6. Transect a $1000-\mu \mathrm{L}$ plastic pipet tip approximately $3 \mathrm{~mm}$ from the end of the tip using a razor blade.

7. Using the transected pipet tip, transfer the agarose/cell mixture to the microfuge tube that was previously filled with agaraose.

8. While the agarose is still melted, add a wooden toothpick to the tube. This facilitates removal of the cells when the agarose is solidified.

9. After the agarose has solidified, remove the cell plug using the embedded toothpick and section the plug in half, generating two cell blocks.

10. Process the agarose plugs into paraffin blocks using standard tissue processing. Several plugs can be placed into a single paraffin block.

\section{Notes}

1. Donor blocks placed under a low wattage bulb will warm up slightly, making them a little softer, less likely to crack, and easier to punch.

2. Because of the limitation in travel along the $x$-axis, to put more than 600 cores into a single TMA block, it is necessary to remove the block from the base plate and rotate the block $180^{\circ}$. If the block is removed, it is difficult to realign the rows and columns.

3. During construction the block will begin to bulge up. Incorporating space between cores minimizes this. Greater spacing will cause less bulging.

4. The use of control tissue is optional, but often quite helpful when examining new and existing immunohistochemical or other markers. In addition, it can serve as a quality control during block construction. For example, once a number of tumor tissues with similar morphologies are placed into a TMA, there is no simple way to check if the tissues were correctly placed. If the array was designed with liver tissue to be placed in position X5,Y3, then the expectation is that the final block will contain such tissue in the proper location. Finding a number of such tissues in place is quite reassuring that the block was indeed correctly constructed.

5. Cut a new H+E before evaluating the blocks to be used. This will ensure that the tissue has not been cut through and that the tissue diagnosis is correct. 
6. Performing immunocytochemistry stains prior to selection of tissue samples will help evaluate the quality of fixation.

7. Practice with the 1.0- or $1.5-\mathrm{mm}$ punch before beginning your array. It is easier to visualize what you are doing with the larger size punches.

8. It may be necessary to use a magnifier during array construction. We use a magnifier with an attached light.

9. It is important to face off the recipient block using a dedicated rotary microtome prior to use. This helps ensure that the block face is smooth and that all the arrays that are made will be in the identical plane. This minimizes the amount of block realignment that will be necessary during sectioning and helps to optimize the number of complete sections that an array block will yield. The backside of the block should also be checked to to ensure that it is level as well.

10. Use the practice block to test out the proper water bath temperature before cutting the first array block.

11. Immunocytochemistry using the capillary gap method will not work with the tape transfer slides, unless paired with a blank capillary gap slide.

12. The Ventana autostainer is not compatible with tape transfer slides; the oil cover slip seems to interfere with the staining process.

13. If the area within the block that is selected contains tissue that is quite thin, it may be advantageous to select an alternate sample. Thin donor blocks will not yield many Tissue Microarray slides. In general, the tissue in the donor block should be at least $1.5 \mathrm{~mm}$ thick, although at times certain types of samples may not contain this much tissue. In this case, one must decide on the relative merits of using a TMA vs simply cutting standard blocks and staining standard slides. For very precious specimens such as brain biopsies we have successfully prepared TMAs using quite thin specimens.

14. Although other types of paraffin can be employed, in our recipient blocks we use Paraplast X-tra, which is softer than standard paraffin. We also use deep molds. Care should be exercised to avoid trapping air bubbles under the cassette.

15. Check all needles on receipt; some arrive bent.

16. The most agility is required while expressing the tissue into the recipient block. A margin of $2.5 \mathrm{~mm}$ of paraffin is recommended around the entire array. This prevents the recipient block from cracking during construction.

17. Overtightening of the screws on the arrayer can damage components. It is necessary to turn them only until they are tight.

18. Adjustments can be made to the positions of the punch in the holder. There are four screws holding the V-block; they all need to be loosened before turning the front to back setscrew. Clockwise turning will bring the punch forward. Counterclockwise turning will bring it backward. Left-to-right alignment is achieved using the setscrews on the left and right sides of the turret.

19. The depth of the recipient core can be set by the depth stop, which is located in the upper left hand corner of the turret. But there is no depth stop incorporated into the design of the Tissue Arrayer that will dictate the depth of the donor core. This depth cannot be the same when you are going from one block to another because 
tissue blocks are of varying thickness, which will determine the limit of the depth of the cores that can be taken. Beecher has recently engineered a Depth Stop Kit, which will aid in extracting reproducible lengths of donor cores. Although an experienced person may not need these, a novice will find them useful.

20. Always return the arms of the needles to their original positions. This ensures that the orientation of all the cores will be reproducible.

21. If the core sticks in a block, rotate the stylus back and forth, this will help free the core from the block. In addition, tamping the top of the stylus will help to remove it.

22. After doing several punches the stylus/punch complex may retain some paraffin. Moving the stylus up and down and wiping it with a Kimwipe will remove the paraffin. Using xylene is not necessary and may damage the punch. Occasionally it is necessary to remove the stylus from the punch and wipe it off.

23. You can make your own depth stop by cutting a small section of a thin transfer pipet to the desired length and placing it at the top of the arraying needle.

24. There is no depth stop to aid in removal of tissue samples from the donor block. This is refined with some practice.

25. Do not push the donor punch all the way into its respective hole; let it protrude slightly. It can then be gently pressed in further with a clean glass slide. This ensures that its position is level with the recipient block surface, not in too deep or protruding out.

26. It is possible to cut down the size of a donor core that is too long. Eject the core from the stylus and place on a clean flat surface. Use a clean razor blade to cut the core to the desired length. The core can than be placed into the recipient block with a pair of forceps. This is easier with the larger size punches.

27. It is possible to remove a wrongly placed core with the donor punch. It may leave some material behind. Make a note of it on the map.

28. Cores that are placed too deeply may be removed with the smaller size needle (recipient needle). An additional core can then be inserted. This process should not be considered routine for several reasons. The hole in the recipient block may become enlarged and some residual tissue will be left behind. Cores placed erroneously may also be removed in this fashion. Be sure to make a note whenever this is necessary because the residual tissue may yield incorrect data.

29. The array block should be wiped, brushed, or dusted after the placement of every punch.

30. It is easiest to move horizontally across the map because the $x$-axis adjustment knob is more accessible.

31. The punches can be sharpened with a fine sharpening stone if they become dull (after thousands of punches).

32. Several accessories have been added to the Beecher product line that are useful and worth mentioning. The Four-Block indexer allows four replicate blocks to be made simultaneously. Extended hours of making tissue microarrays can lead to wrist fatigue and aggravate carpal tunnel syndrome. The Motorized Positioner for the Manual Arrayer can alleviate this. 
33. To avoid damaging the array block when you take it from the $37^{\circ} \mathrm{C}$ oven, practice with a couple of empty recipient blocks to familiarize yourself on how the block/ slide complex feels after warming.

34. Also, make sure the section is placed parallel with the slide edge. Having the sections in the proper orientation on the slide greatly facilitates scanning of the slide with automated slide scanners.

35. Recently developed specialized tools are available to help align the block in the microtome to minimize tissue loss, although they are not compatible with all microtomes (http://www.microm.de/products-accessories-Histo.htm).

36. When sectioning a TMA block, we typically cut 20 sections at a time. No published reports on the best methods of storing TMA slides are available. While some are stored under nitrogen gas, we store unstained sections without baking the slides and at $-20^{\circ} \mathrm{C}$. We have had good success with this method with several antibodies over several months' time. However, the long-term effects of this storage method have not been examined.

\section{Acknowledgments}

We would like to thank Cellie Southerland for her diligence and expertise in TMA construction and for providing us with a large number of useful insights for this manuscript. We also thank Don Vindivich for expertise and help in preparing Fig. 2.

This work was funded by Public Health Services Specialized Program in Research Excellence (SPORE) in Prostate Cancer grant no. P50CA58236, The CaPCURE Foundation, and The Lee Family Foundation.

\section{References}

1 Battifora, H. (1986) The multitumor (sausage) tissue block: Novel method for immunohistochemical antibody testing. Lab. Invest. 55, 244-248.

2 Kononen, J., Bubendorf, L., Kallioniemi, A., et al. (1998) Tissue microarrays for high-throughput molecular profiling of tumor specimens. Nat. Med. 4, 844-847.

3 Moch, H., Kononen, T., Kallioniemi, O. P., and Sauter, G. (2001) Tissue microarrays: What will they bring to molecular and anatomic pathology? Adv. Anat. Pathol. 8, 14-20.

4 Bubendorf, L., Nocito, A., Moch, H., and Sauter, G. (2001) Tissue microarray (TMA) technology: Miniaturized pathology archives for high-throughput in situ studies. J. Pathol. 195, 72-79.

5 Rimm, D. L., Camp, R. L., Charette, L. A., Olsen, D. A., and Provost, E. (2001) Amplification of tissue by construction of tissue microarrays. Exp. Mol. Pathol. 70, 255-264.

6 Rimm, D. L., Camp, R. L., Charette, L. A., Costa, J., Olsen, D. A., and Reiss, M. (2001) Tissue microarray: A new technology for amplification of tissue resources. Cancer J. 7, 24-31. 
7. Jensen, T. A. and Hammand, M. E. H. (2001) The tissue microarray-a technical guide for histologists. J. Histotechnol. 24, 283-287.

8 Camp, R. L., Charette, L. A., and Rimm, D. L. (2000) Validation of tissue microarray technology in breast carcinoma. Lab. Invest. 80, 1943-1949.

9 Torhorst, J., Bucher, C., Kononen, J., et al. (2001) Tissue microarrays for rapid linking of molecular changes to clinical endpoints. Am. J. Pathol. 159, 2249-2256.

10 Rubin, M., Dunn, R., Strawderman, M., and Pienta, K. J. (2002) Tissue microarray sampling strategy for prostate cancer biomarker analysis. Am. J. Surg. Pathol. 26, 312-319.

11 Nocito, A., Bubendorf, L., Maria Tinner, E., et al. (2001) Microarrays of bladder cancer tissue are highly representative of proliferation index and histological grade. J. Pathol. 194, 349-357.

12 Hoos, A., Urist, M. J., Stojadinovic, A., et al. (2001) Validation of tissue microarrays for immunohistochemical profiling of cancer specimens using the example of human fibroblastic tumors. Am. J. Pathol. 158, 1245-1251.

13 Meeker, A. K., Gage, W. R., Hicks, J. L., et al. (2002) Telomere length assessment in human archival tissues: Combined telomere fluorescence in situ hybridization and immunostaining. Am. J. Pathol. 160, 1259-1268.

14 Manley, S., Mucci, N. R., De Marzo, A. M., and Rubin, M. A. (2001) Relational database structure to manage high-density tissue microarray data and images for pathology studies focusing on clinical outcome: The prostate specialized program of research excellence model. Am. J. Pathol. 159, 837-843.

15 Liu, C. L., Prapong, W., Natkunam, Y., et al. (2002) Software tools for highthroughput analysis and archiving of immunohistochemistry staining data obtained with tissue microarrays. Am. J. Pathol. 161, 1557-1565.

16. Camp, R. L., Chung, G. G., and Rimm, D. L. (2002) Automated subcellular localization and quantification of protein expression in tissue microarrays. Nat. Med. 8, 1323-1327. 
